\title{
Zymography of Hybrid Layers Created Using Extrafibrillar Demineralization
}

Journal of Dental Research 2018, Vol. 97(4) 409-4I5

(C) International \& American Associations for Dental Research 2017 Reprints and permissions: sagepub.com/journalsPermissions.nav DOI: I0.1 I 77/00220345|7747264 journals.sagepub.com/home/jdr

\author{
L. Gu', A. Mazzoni ${ }^{2}$, Y. Gou ${ }^{3}$, C. Pucci $^{4}$, L. Breschi ${ }^{2}$, D.H. Pashley ${ }^{5}$, \\ L. Niu ${ }^{6}$, and F.R. Tay
}

\begin{abstract}
A chelate-and-rinse extrafibrillar calcium chelation dentin bonding concept has recently been developed and investigated for its effectiveness in improving resin-dentin bonding by bridging the gap between wet and dry dentin bonding. The objective of the present study was to evaluate the gelatinolytic activity of hybrid layers (HLs) created using the chelate-and-rinse bonding technique. Gelatinolytic activity within the $\mathrm{HL}$ was examined using in situ zymography and confocal laser-scanning microscopy after 24-h storage or after thermomechanical cycling. Dentin specimens were bonded with Prime\&Bond NT (Dentsply Sirona) after conditioning with 15 wt\% phosphoric acid for $15 \mathrm{~s}$ (control) or $15 \mathrm{wt} \%$ polymeric chelators (sodium salt of polyacrylic acid; PAAN) of 2 different molecular weights for $60 \mathrm{~s}$. For each reagent, bonding was performed using dry-bonding and wet-bonding techniques $(n=10)$. Slices containing the adhesive-dentin interface were covered with fluorescein-conjugated gelatin and examined with a confocal laser-scanning microscope. Fluorescence intensity emitted by the hydrolyzed fluorescein-conjugated gelatin was quantified. Gelatinolytic activity was expressed as the percentage of green fluorescence emitted within the HL. After storage for $24 \mathrm{~h}$, enzymatic activity was only detected within the completely demineralized phosphoric acid-etched dentin, with values derived from dry bonding higher than those from wet bonding $(P<0.05)$. Almost no fluorescence signals were detected within the $\mathrm{HL}$ when dentin was conditioned with PAANs compared with the controls $(P<0.05)$. After thermomechanical cycling, enzymatic activities significantly increased for the phosphoric acid-conditioned, drying-bonding group compared with 24 -h storage $(P<0.05)$. The present study showed that the use of the chelate-and-rinse bonding concept for both dry-bonding and wet-bonding approaches results in the near absence of matrix-bound collagenolytic activities in the $\mathrm{HL}$ even after aging. This may be attributed to fossilization of endogenous proteases via preservation of intrafibrillar minerals within the dentin collagen matrix.
\end{abstract}

Keywords: bonding, chelation, dentin, matrix metalloproteinases, enzymology, microscopy

\section{Introduction}

Despite significant advancements in adhesive dentistry over the past decade, the durability of resin-dentin bonds remains a formidable issue that has not been satisfactorily addressed (Mazzoni et al. 2017). In vivo and in vitro studies showed that the hybrid layers (HLs) created in dentin by adhesives are unstable in aqueous environments due to hydrolysis of resin components (Tjäderhane et al. 2013a) and degradation of collagen matrices via endogenous proteases such as matrix metalloproteinases (MMPs) and cysteine cathepsins (Mazzoni et al. 2015). To prolong the durability of resin-dentin bonds, inactivation of these proteases has been recommended using synthetic MMP inhibitors (Breschi, Martin, et al. 2010; Breschi, Mazzoni, et al. 2010; Liu et al. 2011; Almahdy et al. 2012), quaternary ammonium methacrylates, or benzalkonium chloride (Tezvergil-Mutluay, Agee, et al. 2011; Tezvergil-Mutluay, Mutluay, et al. 2011). Ethanol wet-bonding, biomimetic remineralization of HLs, and the use of collagen cross-linking agents have also been proposed to increase the longevity of resindentin bonds (Tay and Pashley 2009; Bedran-Russo et al. 2010; Tjäderhane et al. 2013b; Bedran-Russo et al. 2014). Although these protocols yielded promising in vitro results, most of them
'Department of Operative Dentistry and Endodontics, Guanghua School of Stomatology \& Guangdong Provincial Key Laboratory of Stomatology, Sun Yat-sen University, Guangzhou, PR China

${ }^{2}$ Department of Biomedical and Neuromotor Sciences, DIBINEM, University of Bologna-Alma Mater Studiorum, Bologna, Italy ${ }^{3}$ State Key Laboratory of Oral Diseases, West China Hospital of Stomatology, Sichuan University, Chengdu, China

${ }^{4}$ Department of Restorative Dentistry, Institute of Science and Technology, São Paulo State University UNESP São Jose dos Campos, São Paulo, Brazil

${ }^{5}$ Department of Endodontics, The Dental College of Georgia, Augusta University, Augusta, GA, USA

${ }^{6}$ State Key Laboratory of Military Stomatology \& National Clinical Research Center for Oral Diseases \& Shaanxi Key Laboratory of Oral Diseases, Department of Prosthodontics, School of Stomatology, The Fourth Military Medical University, Xi'an, Shaanxi, PR China

A supplemental appendix to this article is available online.

\section{Corresponding Authors:}

L. Niu, School of Stomatology, The Fourth Military Medical University, 145 West Changle Road, Xi'an 710032, China.

Email: niulina831013@I26.com

F.R. Tay, Department of Endodontics, the Dental College of Georgia, Augutsa University, I 430 John Wesley Gilbert Drive, Augusta, GA 309/2-I I29, USA. Email: ftay@augusta.edu 
require additional steps that render dentin bonding more complicated. To date, easily achieved and clinically feasible techniques to improve long-term dentin bonding are lacking.

In mineralized dentin, MMPs are embedded within the collagen matrix by intrafibrillar apatite crystallites, rendering the proteases immobile and nonfunctional (Pashley et al. 2004). As long as collagen fibrils in dentin are mineralized, the proteases remain structurally stable and inactive (Nishitani et al. 2006). Conversely, depletion of intrafibrillar minerals by acidic conditioners during dentin bonding releases and activates the proforms of endogenous proteases within the water-rich collagen matrix. This, in turn, triggers progressive proteolytic degradation of the exposed collagen fibrils within the HLs (Frassetto et al. 2016).

Recently, a chelate-and-rinse conditioning technique based on extrafibrillar collagen demineralization has been proposed, using a polymeric chelator (sodium salt of poly(acrylic) acid, PAAN) to selectively demineralize extrafibrillar dentin (Mai et al. 2017). This unique bonding concept is based on the size exclusion characteristics of fibrillar collagen; molecules larger than $40 \mathrm{kDa}$ are prevented from accessing the intrafibrillar water compartments of collagen fibrils (Toroian et al. 2007). By using polymeric chelators that are larger than $40 \mathrm{kDa}$, dentin demineralization is confined exclusively to the extrafibrillar spaces. Preservation of intrafibrillar minerals prevents collapse of the partially demineralized dentin matrix when PAAN-conditioned dentin is rinsed and air-dried prior to application of etch-and-rinse adhesives. The technique potentially eliminates problems associated with water-wet dentin bonding (Spencer and Wang 2002). The chelate-and-rinse concept also prevents exposure of endogenous proteases by preserving intrafibrillar minerals in the dentin matrix. Because the proteases are fossilized by intrafibrillar apatite and no mineral-free collagen fibrils are generated by Brønsted-Lowry acidic conditioners (Stoyanov et al. 2010), this should enhance the longevity of resin-dentin bonds.

Accordingly, the objective of the present study was to evaluate the gelatinolytic activity of resin-dentin interfaces created by the extrafibrillar demineralization-based chelate-and-rinse technique, using in situ zymography (Mazzoni et al. 2012) and functional enzyme activity assays. The null hypotheses tested were as follows: 1) HLs created with the chelate-and-rinse technique are not affected by endogenous proteases, regardless of whether dentin is bonded using wet bonding or dry bonding; 2) proteolytic activities within the HLs created by both the chelate-and-rinse and etch-and-rinse techniques do not increase following aging; and 3) PAAN, as a chelator of $\mathrm{Ca}^{2+}$, has no inhibitory effect on the activities of $\mathrm{Ca}^{2+}$-dependent MMP-9 and cathepsin $\mathrm{K}$.

\section{Materials and Methods}

\section{In Situ Zymography}

Sixty extracted intact, noncarious human third molars were obtained according to a protocol approved by the Human Assurance Committee of Augusta University. The teeth were stored in $0.9 \%(\mathrm{w} / \mathrm{v}) \mathrm{NaCl}$ containing $0.02 \%$ sodium azide at $4^{\circ} \mathrm{C}$ for no longer than $1 \mathrm{wk}$. A 1 -mm-thick tooth segment was obtained from the mid-coronal portion of each tooth, using a slow-speed diamond-impregnated saw (Isomet; Buehler) with water cooling. The coronal surface of each tooth segment was polished with 600-grit wet silicon carbide paper (CarbiMet PSA; Buehler) for $1 \mathrm{~min}$ to create a standardized smear layer.

Commercially available acids were diluted in deionized water to produce the concentrations required for their use as dentin conditioners: $15 \mathrm{wt} \%$ phosphoric acid (MilliporeSigma) and $15 \mathrm{wt} \%$ PAAN (average molecular weight 60,000 and 225,000 [Polysciences]; designated as PAAN $_{60 \mathrm{~K}}$ and PAAN $_{225 \mathrm{~K}}$ ). The 2 PAANs were selected because their molecular weights are larger than the 6 - to $40-\mathrm{kDa}$ range for molecules to be partially or completely retained within the intrafibrillar water compartments of demineralized collagen (Toroian et al. 2007). Based on the authors' previous work (Mai et al. 2017; Appendix Fig. 1), the microtensile bond strengths of commercially available 2-step etch-and-rinse adhesives to human dentin conditioned with $15 \mathrm{wt} \%$ PAANs are comparable with that achieved using $15 \mathrm{wt} \%$ phosphoric acid. The latter represents the clinical standard for conditioning dentin using the etch-and-rinse technique. The $15 \mathrm{wt} \%$ PAANs were used herein for examining the effects of the chelate-and-rinse conditioning technique on enzymatic activities within the HLs.

Dentin conditioning was performed by applying the respective etchant liberally to a polished dentin surface and agitating the surface with a microbrush. A 2-step etch-and-rinse adhesive (Prime\&Bond NT; Dentsply Sirona) was applied on dentin conditioned with $15 \mathrm{wt} \%$ phosphoric acid for $15 \mathrm{~s}$ (control), 15 wt $\%$ PAAN $_{60 \mathrm{~K}}$ for $60 \mathrm{~s}$, or $15 \mathrm{wt} \%$ PAAN $_{225 \mathrm{~K}}$ for $60 \mathrm{~s}$. Six experimental groups were created by applying the adhesive using the dry-bonding or the wet-bonding technique. Sixty tooth segments were randomly assigned to each treatment group, with 10 segments per group. For dry bonding, conditioned dentin was air-dried with oil- and moisture-free air for $5 \mathrm{~s}$. For wet bonding, conditioned dentin was kept moist by blotting excess water with lint-free tissues. Prime\&Bond NT was subsequently applied to the conditioned dentin for $20 \mathrm{~s}$, gently air-dried for $5 \mathrm{~s}$, and light-cured for $10 \mathrm{~s}$ according to the manufacturer's instructions. A 1-mm-thick flowable composite (Protect Liner F; Kuraray Noritake) was applied to the bonded disks and lightcured for $20 \mathrm{~s}$ using a quartz-tungsten-halogen light-curing unit (Curing Light 2500; 3M ESPE). After bonding, specimens from each group were divided into 2 subgroups $(n=5)$. One subgroup (designated as $\mathrm{T}_{0}$ ) was stored in deionized water at $37^{\circ} \mathrm{C}$ for $24 \mathrm{~h}$. The other subgroup (designated as $\mathrm{T}_{1}$ ) was thermomechanically challenged, using 10,000 thermal cycles $\left(10^{\circ} \mathrm{C}\right.$, $25^{\circ} \mathrm{C}$, and $55^{\circ} \mathrm{C}$ for $1 \mathrm{~min}$ each) and 240,000 mechanical cycles, corresponding to $1 \mathrm{y}$ of intraoral use (Perote et al. 2015). Each bonded specimen was sectioned vertically into 1-mm-thick slabs to expose the resin-dentin interface using the Isomet saw. The 2 central slabs of each tooth were selected, yielding 10 slabs per subgroup for microscopy $(n=10)$.

Each bonded slab was affixed to a glass slide with cyanoacrylate cement (Loctite super glue; Henkel) and serially polished with 600- and 1,200-grit wet silicon carbide papers (MicroCut PSA; Buehler) to obtain a $\sim 50-\mu \mathrm{m}$-thick section. 
Final polishing was achieved using 4,000-grit wet silicon carbide paper (MicroCut S-P400; Buehler) for 5 min to obtain a glossy surface for microscopic evaluation. In situ zymography was performed with quenched fluorescein-conjugated gelatin (E-12055; Molecular Probes) as the MMP substrate (Mazzoni et al. 2012). Briefly, $50 \mu \mathrm{L}$ of the fluorescent gelatin mixture was placed on top of each slab and protected with a cover slip. Slides were light protected and incubated in a $100 \%$ relative humidity chamber at $37^{\circ} \mathrm{C}$ for $48 \mathrm{~h}$. Hydrolysis of the quenched fluorescein-conjugated gelatin, which is indicative of endogenous gelatinolytic activity, was evaluated with a confocal laserscanning microscope (excitation/emission: $488 / 530 \mathrm{~nm}$; LSM 780; Carl Zeiss). For each specimen, 3 areas of consistent dimensions, located at the midpoint between the 2 enameldentin junctions, were selected for gelatinolytic activity evaluation. For each area, 20 optical sections (350 nm thick) were acquired from different focal planes. Stacked images were processed with the ZEN 2010 software (Carl Zeiss). Microscopy was performed by an independent observer who was unaware of the treatment applied to the tooth segments. Fluorescence intensity emitted by the hydrolyzed fluorescence-conjugated gelatin was quantified using ImageJ software (National Institutes of Health). Gelatinolytic activity was expressed as a percentage of the green fluorescence within the $\operatorname{HL}(n=3$ areas $\times$ 10 specimens $=30$ ). Because the normality and homoscedasticity assumptions of the data appeared to be valid, a 3-factor analysis of variance (ANOVA) was employed to examine the effects of dentin conditioner, bonding technique, and storage time on gelatinolytic activity. The interaction among the 3 variables on the percentages of HL exhibiting gelatinolytic activities was also evaluated. Post hoc pairwise comparisons were performed using the Tukey test. For all analyses, statistical significance was preset at $\alpha=0.05$ (SPSS 19; SPSS).

\section{Inhibition of Soluble rhMMP9 and Cathepsin $K$ by PAANs}

Both PAAN $_{60 \mathrm{~K}}$ and PAAN $\mathrm{P}_{25 \mathrm{~K}}$ were diluted in deionized water to achieve the required concentrations $(5,10,15,20 \mathrm{wt} \%)$. Determination of whether PAANs inhibit MMP-9 and cathepsin $\mathrm{K}$ was achieved using purified recombinant human (rh) MMP-9, the SensoLyte Generic MMP assay kit, and SensoLyte cathepsin K assay kit (all from AnaSpec). The MMP assay kit contains a thiopeptide that is cleaved by MMPs to release a sulfhydryl group that reacts with 5,5'-dithiobis (2-nitrobenzoic acid) to produce a colored product (2-nitro-5-thiobenzoic acid); the latter was quantitated in a 96-well plate at $412 \mathrm{~nm}$. The cathepsin K kit contains QXL 520/HiLyte-Fluor 488 FRET peptide substrate for specific enzymes. When the FRET substrate is cleaved by a specific enzyme, it releases HiLyte-Fluor 488 fluorescence, which may be monitored at excitation/emission $490 / 520 \mathrm{~nm}$. The potential of PAAN in inhibiting MMP-9 and cathepsin K over time was examined using PAAN at various concentrations. All analyses were performed in quadruplicate according to the manufacturer's instructions. Because the data were normally distributed and homoscedastic, they were analyzed with 1-factor ANOVA and Tukey's test at $\alpha=0.05$.

\section{Results}

\section{In Situ Zymography}

Representative images of dentin conditioned with phosphoric acid or polymeric chelators (PAAN) are presented in Figures 1 to 4. Percentages of HLs exhibiting hydrolysis of the quenched fluorescein-conjugated gelatin in the 6 subgroups are shown in the Appendix Table.

For control specimens bonded after $15 \mathrm{wt} \%$ phosphoric acid etching, in situ zymography revealed green fluorescence within the 5- to $6-\mu \mathrm{m}$-thick HL after $48 \mathrm{~h}$ of incubation. The results were indicative of extensive hydrolysis of the fluorescein-conjugated gelatin at those sites (Fig. 1). Superimposition of the fluorescence image over the differential interference contrast (DIC) image showed that fluorescence was prominent along the entire depth of demineralization in the dry-bonded specimen (Fig. 1C). For the corresponding wet-bonded specimen, fluorescence was intermittent and was most intense within the dentinal tubules and at the bottom of the HL (Fig. 1F). In contrast, no green fluorescence was detected in the PAAN ${ }_{225 \mathrm{k}}$-conditioned HLs at $\mathrm{T}_{0}$, irrespective of whether the dry-bonding or wet-bonding technique was used (Fig. 2). Acquired images in the green channel showed that fluorescence was exclusively located within the dentinal tubules. Similar features were identified for PAAN $_{60 \mathrm{k}}$ (Appendix Fig. 2).

Fluorescence intensity increased after thermomechanical cycling $\left(\mathrm{T}_{1}\right)$ in HLs created with phosphoric acid etching (Fig. 3; Appendix Table). Only resin-dentin interfaces bonded with dry-bonding technique demonstrated significantly higher $(P<$ 0.001 ) fluorescence intensity within the HLs compared to $T_{0}$ (Figs. 1A, C, 3A, C; Appendix Table). For the PAAN groups, fluorescence remained undetectable within HLs created by wet bonding or dry bonding at $\mathrm{T}_{1}$ (Fig. 4; Appendix Fig. 3).

\section{Inhibition of Soluble rhMMP-9 and Cathepsin K}

The effects of PAANs on soluble rhMMP-9 and cathepsin K are represented in Figure 5. The relative percentages of rhMMP-9 and cathepsin $\mathrm{K}$ inhibition by the inhibitor control were $96.3 \%$ $\pm 4.2 \%$ and $77.5 \% \pm 0.1 \%$, respectively. Over $80 \%$ of the rhMMP-9 was inhibited by $15 \mathrm{wt} \%$ PAANs. The extent of inhibition increased in a dose-dependent manner $(P=0.006$ for PAAN $_{60 \mathrm{~K}} ; P<0.001$ for PAAN ${ }_{225 \mathrm{~K}}$ ). Unlike MMP-9, there was negligible inhibition of cathepsin $\mathrm{K}$ at PAAN concentrations of $5 \mathrm{wt} \%$ for both PAAN $_{225 \mathrm{k}}$ and PAAN ${ }_{60 \mathrm{k}}$. Inhibition of cathepsin $\mathrm{K}$ occurred after the enzyme was incubated with increasing PAAN concentrations, reaching $41.1 \%$ inhibition at $20 \mathrm{wt} \%$ PAAN $_{60 K}$. No significant difference was found between the inhibitory effect of PAAN ${ }_{60 K}$ and PAAN $_{225 K}$ at various concentrations ( $P=0.362$ for rhMMP9; $P=0.081$ for cathepsin $\mathrm{K})$.

\section{Discussion}

Differential extrafibrillar demineralization has been proposed as a means for preventing collapse of denuded dentin collagen by air-drying. This may be achieved either by using polymeric chelators with molecular weight $>40 \mathrm{kDa}$ or by using 


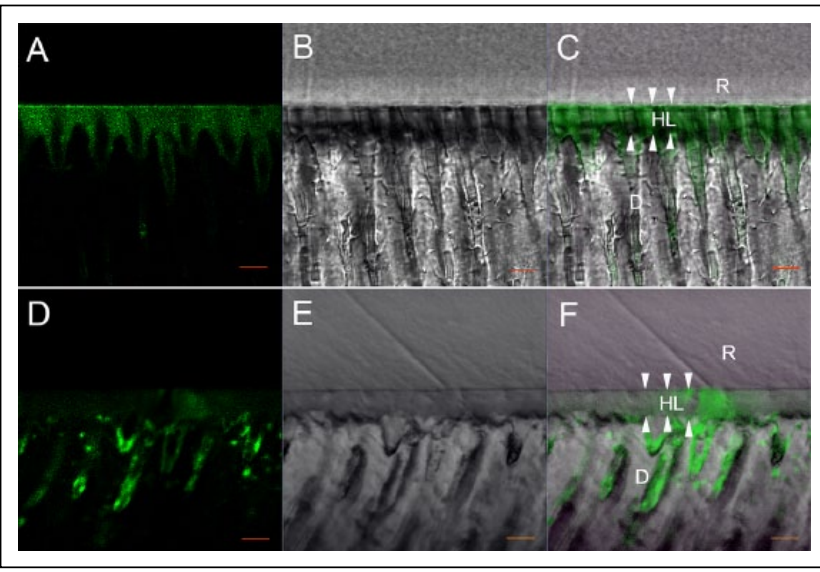

Figure I. Resin-dentin interface conditioned with 15\% phosphoric acid for $15 \mathrm{~s}$, with adhesive bonded using the dry-bonding (A-C) or the wet-bonding (D-F) technique after storage for $24 \mathrm{~h}\left(\mathrm{~T}_{0}\right)$. Specimens were incubated for $48 \mathrm{~h}$ with quenched fluorescence-labeled gelatin. $D$, dentin; HL, hybrid layer (between arrowheads); $R$, adhesive resin. Scale bar, $5 \mu \mathrm{m}$. (A) Confocal image of in situ zymogram acquired in the green channel showing fluorescence (identifying intense endogenous enzymatic activity) within $\mathrm{HL}$ and the dentinal tubules. (B) Differential interference contrast (DIC) image showing the optical density of the resin-dentin interface in the dry-bonded specimen. (C) Merged images $A$ and $B$. (D) Confocal image in the green channel, showing fluorescence at the bottom of the $\mathrm{HL}$ and within the dentinal tubules. (E) DIC image showing the optical density of the resin-dentin interface in the wetbonded specimens. (F) Merged images D and E.

biocompatible chelating agents of any molecular size (Mai et al. 2017). The first protocol completely removes extrafibrillar minerals and creates wide interfibrillar channels for diffusion of resin monomers (Osorio et al. 2010), but it is very time-consuming (20 $\mathrm{min})$. The second protocol is faster but is extremely difficult to fine-tune the application time for small chelating agents $<40 \mathrm{kDa}$ (Mai et al. 2017). In the present study, the 2 extrafibrillar demineralization protocols were combined by conditioning mineralized dentin with polymeric chelating agents $(>40 \mathrm{kDa})$ for $60 \mathrm{~s}$ instead of $30 \mathrm{~s}$ to harness the advantages of both protocols (Mai et al. 2017).

In situ zymography of dentin HLs was developed by Mazzoni et al. (2012) for screening the proteolytic activity of endogenous enzymes directly on the resin-dentin interface. In the present study, fluorescence was absent in HLs created with the chelate-and-rinse conditioning technique. Thus, the first null hypothesis cannot be rejected. More significantly, enzymatic activity of PAAN-conditioned resin-dentin interface created by wet bonding or dry bonding remained undetectable after thermomechanical cycling. In contrast, phosphoric acidetched dentin bonded with dry bonding showed a 2-fold increase in gelatinolytic activity after thermomechanical cycling $(P<0.001)$, whereas the gelatinolytic activity produced by wet bonding remained relatively stable $(P=0.250)$. Accordingly, the second null hypothesis can only be partially rejected.

Proteolytic activities had previously been identified in HLs created with etch-and-rinse and self-etch adhesives (Mazzoni et al. 2006; Nishitani et al. 2006; Mazzoni et al. 2012, 2013). These findings are confirmed in the present study using in situ

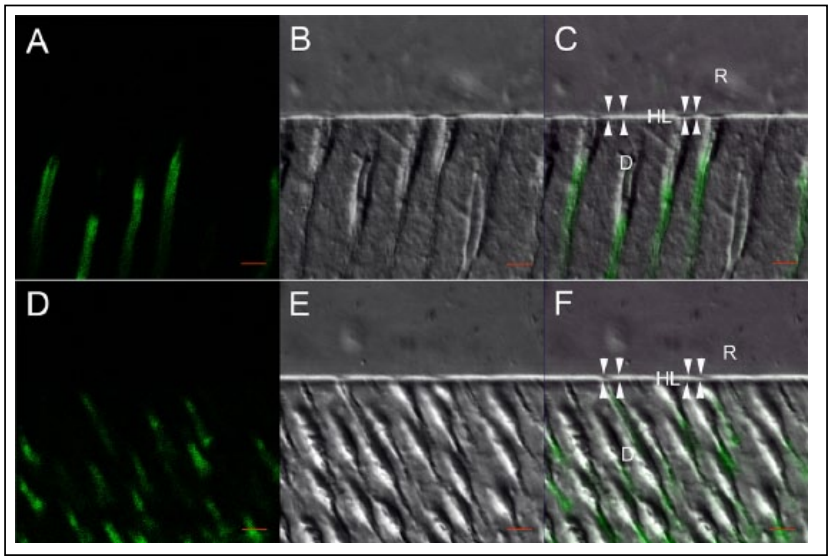

Figure 2. Resin-dentin interface conditioned with 15 wt\% sodium salt of polyacrylic acid (PAAN) (similar features for the 2 PAANs of different molecular weights; Mw 225,000 shown as representative) for $60 \mathrm{~s}$ and prepared using the dry-bonding (A-C) or the wet-bonding (D-F) technique after storage for $24 \mathrm{~h}\left(\mathrm{~T}_{0}\right)$. Specimens were incubated for $48 \mathrm{~h}$ with quenched fluorescein-labeled gelatin. $D$, dentin; $H L$, hybrid layer (between arrowheads); R, adhesive resin. Scale bar, $5 \mu \mathrm{m}$ (A) Confocal image of in situ zymogram acquired in the green channel showing fluorescence within the dentinal tubules only. (B) Differential interference contrast (DIC) image showing the optical density of the resin-dentin interface in the dry-bonded specimen. (C) Merged images $A$ and $B$. (D) Confocal image in the green channel, showing fluorescence within the dentinal tubules only. (E) DIC image showing the optical density of the interface resin-dentin in the wet-bonded specimens. $(F)$ Merged images $D$ and $E$. Absence of fluorescence signals within the $\mathrm{HL}$ was universally observed at $\mathrm{T}_{0}$ using the PAAN, irrespective of the bonding technique employed.

zymography. The location in which proteolytic activity occurred in phosphoric acid-etched, wet-bonded specimens probably represents regions with poorly resin-infiltrated demineralized collagen at the base of HLs. Endogenous proteolytic enzymes localized within HLs are exposed and activated in low-pH environments by inducing the cysteine switch (Sulkala et al. 2001; Chaussain-Miller et al. 2006; Tersariol et al. 2010). These proteases are responsible for degradation of denuded collagen fibrils within the HLs and are important contributors to the poor durability of resin-dentin bonds. Gelatinolytic activities present in dentinal tubules of mineralized dentin are predominantly identified along the periphery of those tubules (Mazzoni et al. 2012). Such activities were thought to be derived from the lamina limitans lining the dentinal tubule walls, where these proteins participate in the regulation of peritubular dentin formation (Hannas et al. 2007). Intratubular gelatinolytic activities may also be caused by precipitation of dentinal fluid-derived MMPs along the tubular wall during laboratory specimen processing (Tjäderhane et al. 2012).

Several factors may have contributed to the lack of endogenous gelatinolytic activity within the HLs created with PAAN. With average molecular weights of 225 and $60 \mathrm{kDa}$, the 2 PAANs tested in the present study were too bulky to access any of the water compartments within the mineralized collagen fibrils (Toroian et al. 2007). The potential of using the polymeric chelator PAAN to produce selective extrafibrillar demineralization along the entire depth of demineralization has been demonstrated previously (Mai et al. 2017). Compared with the 


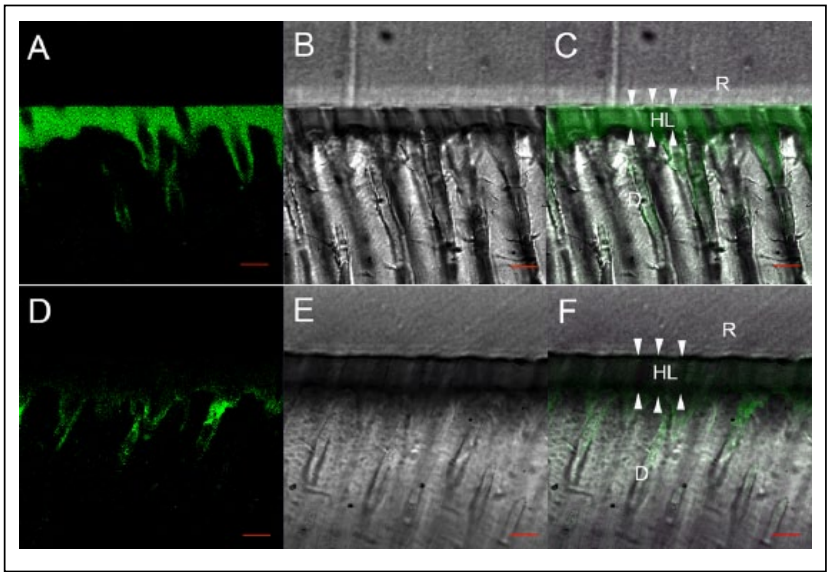

Figure 3. Resin-dentin interface conditioned with $15 \%$ phosphoric acid for $15 \mathrm{~s}$ and bonded using the dry-bonding (A-C) or wet-bonding (D-F) technique after thermomechanical cycling $\left(T_{1}\right)$. Specimens were incubated for $48 \mathrm{~h}$ with quenched fluorescein-labeled gelatin. D, dentin; $H L$, hybrid layer (between arrowheads); R, adhesive resin. Scale bars, $5 \mu \mathrm{m}$. (A) Confocal image of in situ zymogram acquired in the green channel showing fluorescence within $\mathrm{HL}$ and the dentinal tubules. (B) Differential interference contrast (DIC) image showing the optical density of the resin-dentin interface in the dry-bonded specimen. (C) Merged images A and B. (D) Confocal image in the green channel showing fluorescence at the bottom of $\mathrm{HL}$ and within the dentinal tubules. (E) DIC image showing the optical density of the interface resindentin in the wet-bonded specimen. (F) Merged images D and E.

2- to 5-nm-wide intrafibrillar spaces, the water-filled interfibrillar spaces are much wider (20 to $30 \mathrm{~nm}$ ) (Liu et al. 2014). By retaining intrafibrillar minerals, dentin collagen matrices conditioned with $15 \mathrm{wt} \%$ PAAN solutions are also more resistant to collapse upon air-drying. This ensues optimal infiltration of resin monomers into the mineral-depleted extrafibrillar spaces even with the use of more bulky resin monomers (Bertassoni et al. 2012). Of particular importance is that gelatinolytic activities of adhesive-treated, PAAN-conditioned dentin were hardly detectable after the application of acidic resin monomers (Prime\&Bond NT, pH 2.59) (Figs. 2, 4). Intrafibrillar minerals retention is pivotal in preventing exposure and activation of endogenous proteases during bonding. The slightly alkaline $\mathrm{pH}$ of $15 \mathrm{wt} \%$ PAAN ( $\mathrm{pH} 8.03-8.26$ ) may also help preserve the activity of tissue inhibitors of MMPs (Mazzoni et al. 2015). These factors may have all contributed to protecting the partially demineralized dentin collagen matrix from enzymatic degradation over time.

The authors were dubious initially whether PAAN, being a chelator of calcium ions, is a potent inhibitor of calcium ionsensitive MMPs and cathepsins. This was the rationale behind the use of assays for evaluating MMP-9 and cathepsin K activities. Results of the quantitative assay indicate that the extent of MMP-9 and cathepsin $\mathrm{K}$ inhibition was proportional to PAAN concentrations. This necessitates rejection of the third null hypothesis. Nevertheless, $15 \mathrm{wt} \%$ PAAN inhibited only $79.8 \%$ to $86.9 \%$ of soluble rhMMP-9 and $22.5 \%$ to $38.2 \%$ of cathep$\sin \mathrm{K}$, which were significantly lower than the results obtained from the respective inhibitor control. Together with the absence of gelatinolytic activity in HLs, these findings indicate that MMPs and cathepsin K could be immobilized by intrafibrillar

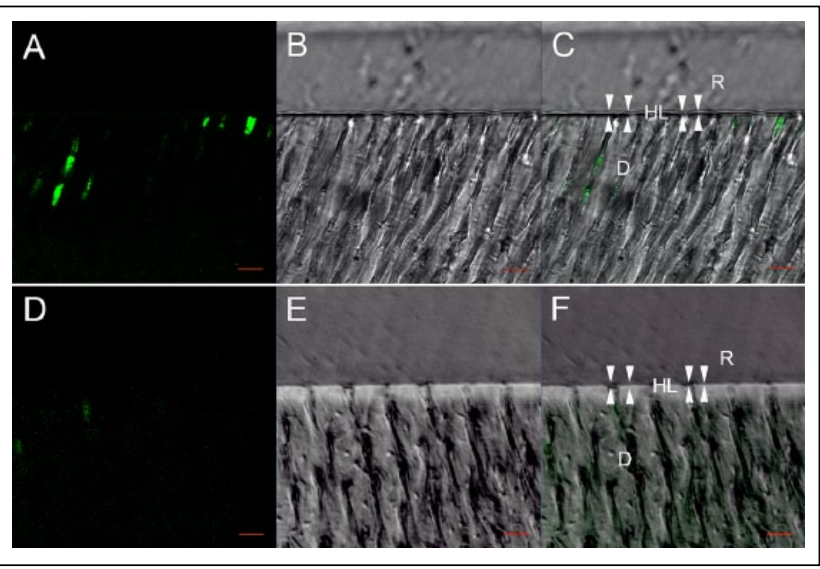

Figure 4. Resin-dentin interface conditioned with 15 wt\% sodium salt of polyacrylic acid (PAAN) (similar features for the 2 PAANs of different molecular weights; Mw 225,000 shown as representative) for $60 \mathrm{~s}$ and prepared using the dry-bonding $(\mathbf{A}-\mathbf{C})$ or wet-bonding $(\mathbf{D}-\mathbf{F})$ technique after thermomechanical cycling $\left(T_{1}\right)$. Specimens were incubated for 48 $h$ with quenched fluorescein-labeled gelatin. $D$, dentin; $H L$, hybrid layer (between arrowheads); R, adhesive resin. Scale bar, $5 \mu \mathrm{m}$. (A) Confocal image of in situ zymogram acquired in the green channel showing fluorescence in dentinal tubules only. (B) Differential interference contrast (DIC) image showing the optical density of the resin-dentin interface in the dry-bonded specimen. (C) Merged images A and B. (D) Confocal image in the green channel exhibited almost no fluorescence. (E) DIC image showing the optical density of the interface resin-dentin in the wet-bonded specimens. (F) Merged images $D$ and $E$. Absence of fluorescence signals within the $\mathrm{HL}$ was universally observed at $\mathrm{T}$, using the PAAN, irrespective of the bonding technique employed.

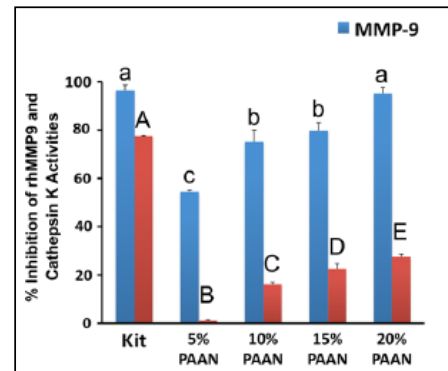

(A) PAAN $225 \%$

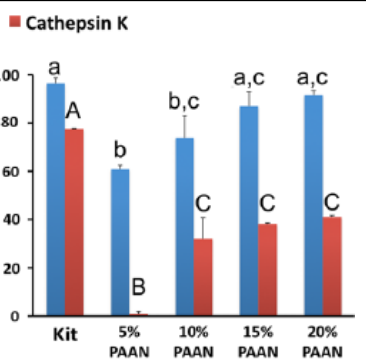

(B) PAAN $60 K$
Figure 5. Percentage inhibition of rhMMP- 9 and cathepsin $\mathrm{K}$ by increasing concentrations of PAANs of different average molecular weights (indicated by subscripts after PAAN), (A) PAAN ${ }_{225 \mathrm{k}}$ and (B) PAAN $_{60 \mathrm{k}}$. Values are means and standard deviations of the percentage inhibition. PAAN concentration is given in weight\%. Groups designated with the same letters (uppercase or lowercase) on top of the bars are not statistically different $(P>0.05)$. Kit, kit inhibitors for MMP-9 and cathepsin K; MMP, matrix metalloproteinase; PAAN, sodium salt of polyacrylic acid.

minerals when the chelate-and-rinse technique was used. The latter probably provides more resistance to bond degradation by proteolytic enzymes over time compared with the use of the etch-and-rinse technique.

Water plays "yin" and "yang" roles in dentin bonding. Water prevents interpeptide hydrogen bonding between completely demineralized collagen fibrils and hence collapse of the demineralized collagen matrix during air-drying (Pashley et al. 2003). Paradoxically, water is also the critical barrier for sustaining 
stable resin-dentin bonds. When water is incompletely replaced during adhesive infiltration, the residual water provides a medium for proteolytic destruction of the denuded collagen (Zhang et al. 2016). Hence, the capability of polymeric chelators with molecular sizes $>40 \mathrm{kDa}$ to retain intrafibrillar minerals represents a window of opportunity for elimination of water-wet bonding. Retention of intrafibrillar minerals implies that fossilized endogenous proteases cannot participate in collagen matrix degradation (Garnero et al. 2003). These factors are conducive to long-term resin-dentin bond integrity.

Within the limitations of the present study, it may be concluded that the use of the extrafibrillar demineralization-based, chelate-and-rinse conditioning technique substantially reduces endogenous proteolytic enzyme-initiated collagen degradation within HLs. Collagen degradation remained stable after thermomechanical cycling. The present study is among the first to demonstrate the potential of employing "selective demineralization" to prevent HL degradation. Future studies are required to validate the feasibility of using selective demineralization and dry bonding with nonacidic, hydrophobic adhesive resin monomers to support the use of this technique in vivo. This should provide more convincing evidence to justify that the chelate-and-rinse bonding technique is an alternative to the etch-and-rinse wet-bonding technique.

\section{Author Contributions}

L. $\mathrm{Gu}$, contributed to data acquisition, drafted and critically revised the manuscript; A. Mazzoni, contributed to design and data analysis, drafted the manuscript; Y. Gou, contributed to data acquisition and analysis, critically revised the manuscript; C. Pucci, contributed to data acquisition and analysis, drafted the manuscript; L. Breschi, contributed to design, data analysis, and interpretation, critically revised the manuscript; D.H. Pashley, contributed to conception, design, data analysis, and interpretation, critically revised the manuscript; L. Niu, F.R. Tay, contributed to conception, design, and data acquisition, drafted and critically revised the manuscript. All authors gave final approval and agree to be accountable for all aspects of the work.

\section{Acknowledgments}

This work was supported by grants 81722015,81200776 , and 81720108011 from National Nature Science Foundation of China; grant 2014J2200099 from the Science and Technology Program of Guangzhou; grant 2016YFC1101400 from the National Key Research and Development Program of China; grant 2015AA0 20942 from the National High Technology Research and Development Program of China; and grant 2016KJXX-18 from Shaanxi New-star Plan of Science and Technology. The authors declare no potential conflicts of interest with respect to the authorship and/or publication of this article.

\section{References}

Almahdy A, Koller G, Sauro S, Bartsch JW, Sherriff M, Watson TF, Banerjee A. 2012. Effects of MMP inhibitors incorporated within dental adhesives. J Dent Res. 91(6):605-611.

Bedran-Russo AK, Pauli GF, Chen SN, McAlpine J, Castellan CS, Phansalkar RS, Aguiar TR, Vidal CM, Napotilano JG, Nam JW, et al. 2014. Dentin biomodification: strategies, renewable resources and clinical applications. Dent Mater. 30(1):62-76.

Bedran-Russo AK, Vidal CM, Dos Santos PH, Castellan CS. 2010. Long-term effect of carbodiimide on dentin matrix and resin-dentin bonds. J Biomed Mater Res B Appl Biomater. 94(1):250-255.

Bertassoni LE, Orgel JP, Antipova O, Swain MV. 2012. The dentin organic matrix: limitations of restorative dentistry hidden on the nanometer scale. Acta Biomater. 8(7):2419-2433.

Breschi L, Martin P, Mazzoni A, Nato F, Carrilho M, Tjäderhane L, Visintini E, Cadenaro M, Tay FR, De Stefano Dorigo E, et al. 2010. Use of a specific MMP-inhibitor (galardin) for preservation of hybrid layer. Dent Mater. 26(6):571-578.

Breschi L, Mazzoni A, Nato F, Carrilho M, Visintini E, Tjaderhane L, Ruggeri A Jr, Tay FR, Dorigo Ede S, Pashley DH. 2010. Chlorhexidine stabilizes the adhesive interface: a 2-year in vitro study. Dent Mater. 26(4):320-325.

Chaussain-Miller C, Fioretti F, Goldberg M, Menashi S. 2006. The role of matrix metalloproteinases (MMPs) in human caries. J Dent Res. 85(1):22-32.

Frassetto A, Breschi L, Turco G, Marchesi G, Di Lenarda R, Tay FR, Pashley DH, Cadenaro M. 2016. Mechanisms of degradation of the hybrid layer in adhesive dentistry and therapeutic agents to improve bond durability: a literature review. Dent Mater. 32(2):e41-e53.

Garnero P, Ferreras M, Karsdal MA, Nicamhlaoibh R, Risteli J, Borel O, Qvist P, Delmas PD, Foged NT, Delaissé JM. 2003. The type I collagen fragments ICTP and CTX reveal distinct enzymatic pathways of bone collagen degradation. J Bone Miner Res. 18(5):859-867.

Hannas AR, Pereira JC, Granjeiro JM, Tjäderhane L. 2007. The role of matrix metalloproteinases in the oral environment. Acta Odontol Scand. 65(1):1-13.

Liu Y, Tjäderhane L, Breschi L, Mazzoni A, Li N, Mao J, Pashley DH, Tay FR. 2011. Limitations in bonding to dentin and experimental strategies to prevent bond degradation. J Dent Res. 90(8):953-968.

Liu Y, Thomopoulos S, Chen C, Birman V, Buehler MJ, Genin GM. 2014. Modelling the mechanics of partially mineralized collagen fibrils, fibres and tissue. J R Soc Interface. 11(92):20130835.

Mai S, Wei CC, Gu LS, Tian FC, Arola DD, Chen JH, Jiao Y, Pashley DH, Niu LN, Tay FR. 2017. Extrafibrillar collagen demineralization-based chelateand-rinse technique bridges the gap between wet and dry dentin bonding. Acta Biomater. 57:435-448.

Mazzoni A, Angeloni V, Sartori N, Duarte S Jr, Maravic T, Tjäderhane L, Pashley DH, Tay FR, Breschi L. 2017. Substantivity of carbodiimide inhibition on dentinal enzyme activity over time. J Dent Res. 96(8):902-908.

Mazzoni A, Nascimento FD, Carrilho M, Tersariol I, Papa V, Tjäderhane L, Di Lenarda R, Tay fR, Pashley DH, Breschi L. 2012. MMP activity in the hybrid layer detected with in situ zymography. J Dent Res. 91(5):467-472.

Mazzoni A, Pashley DH, Nishitani Y, Breschi L, Mannello F, Tjäderhane L, Toledano M, Pashley EL, Tay FR. 2006. Reactivation of inactivated endogenous proteolytic activities in phosphoric acid-etched dentine by etch-andrinse adhesives. Biomaterials. 27(25):4470-4476.

Mazzoni A, Scaffa P, Carrilho M, Tjäderhane L, Di Lenarda R, Polimeni A, Tezvergil-Mutluay A, Tay FR, Pashley DH, Breschi L. 2013. Effects of etch-and-rinse and self-etch adhesives on dentin MMP-2 and MMP-9. J Dent Res. 92(1):82-86.

Mazzoni A, Tjäderhane L, Checchi V, Di Lenarda R, Salo T, Tay FR, Pashley DH, Breschi L. 2015. Role of dentin MMPs in caries progression and bond stability. J Dent Res. 94(2):241-251.

Nishitani Y, Yoshiyama M, Wadgaonkar B, Breschi L, Mannello F, Mazzoni A, Carvalho RM, Tjäderhane L, Tay FR, Pashley DH. 2006. Activation of gelatinolytic/collagenolytic activity in dentin by self-etching adhesives. Eur J Oral Sci. 114(2):160-166.

Osorio E, Toledano M, Aguilera FS, Tay FR, Osorio R. 2010. Ethanol wet-bonding technique sensitivity assessed by AFM. J Dent Res. 89(11):1264-1269.

Pashley DH, Agee KA, Carvalho RM, Lee KW, Tay FR, Callison TE. 2003. Effects of water and water-free polar solvents on the tensile properties of demineralized dentin. Dent Mater. 19(5):347-352.

Pashley DH, Tay FR, Yiu C, Hashimoto M, Breschi L, Carvalho RM, Ito S. 2004. Collagen degradation by host-derived enzymes during aging. J Dent Res. 83(3):216-221.

Perote LC, Kamozaki MB, Gutierrez NC, Tay FR, Pucci CR. 2015. Effect of matrix metalloproteinase-inhibiting solutions and aging methods on dentin bond strength. J Adhes Dent. 17(4):347-352.

Spencer P, Wang Y. 2002. Adhesive phase separation at the dentin interface under wet bonding conditions. J Biomed Mater Res. 62(3):447-456.

Stoyanov ES, Stoyanova IV, Reed CA. 2010. The structure of the hydrogen ion $(\mathrm{H}(\mathrm{aq})+)$ in water. J Am Chem Soc. 132(5):1484-1485

Sulkala M, Wahlgren J, Larmas M, Sorsa T, Teronen O, Salo T, Tjäderhane L. 2001. The effects of MMP inhibitors on human salivary MMP activity and caries progression in rats. J Dent Res. 80(6):1545-1549. 
Tay FR, Pashley DH. 2009. Biomimetic remineralization of resin-bonded acidetched dentin. J Dent Res. 88(8):719-724.

Tersariol IL, Geraldeli S, Minciotti CL, Nascimento FD, Pääkkönen V, Martins MT, Carrilho MR, Pashley DH, Tay FR, Salo T, et al. 2010. Cysteine cathepsins in human dentin-pulp complex. J Dent. 36(3):475-481.

Tezvergil-Mutluay A, Agee KA, Uchiyama T, Imazato S, Mutluay MM, Cadenaro M, Breschi L, Nishitani Y, Tay FR, Pashley DH. 2011. The inhibitory effects of quaternary ammonium methacrylates on soluble and matrix-bound MMPs. J Dent Res. 90(4):535-540.

Tezvergil-Mutluay A, Mutluay MM, Gu LS, Zhang K, Agee KA, Carvalho RM, Tay FR, Breschi L, Suh BI, Pashley DH. 2011. The anti-MMP activity of benzalkonium chloride. J Dent. 39(1):57-64.

Tjäderhane L, Carrilho MR, Breschi L, Tay FR, Pashley DH. 2012. Dentin basic structure and composition: an overview. Endod Topics. 20(1):3-29.
Tjäderhane L, Nascimento FD, Breschi L, Mazzoni A, Tersariol IL, Geraldeli S, Tezvergil-Mutluay A, Carrilho RM, Tay FR, Pashley DH. 2013a. Strategies to prevent hydrolytic degradation of the hybrid layer: a review. Dent Mater. 29(10):999-1011.

Tjäderhane L, Nascimento FD, Breschi L, Mazzoni A, Tersariol IL, Geraldeli S, Tezvergil-Mutluay A, Carrilho MR, Carvalho RM, Tay FR, et al. 2013b. Optimizing dentin bond durability: control of collagen degradation by matrix metalloproteinases and cysteine cathepsins. Dent Mater. 29(1):116-135.

Toroian D, Lim JE, Price PA. 2007. The size exclusion characteristics of type I collagen: implications for the role of noncollagenous bone constituents in mineralization. J Biol Chem. 282(31):22437-22447.

Zhang ZY, Tian FC, Niu LN, Ochala K, Chen C, Fu BP, Wang XY, Pashley DH, Tay FR. 2016. Defying ageing: an expectation for dentine bonding with universal adhesives? J Dent. 45:43-52. 